\title{
LES COMPÉTENCES DES ADULTES À LA LOUPE \#2 2016
}

\section{Qu'entend-on réellement par faibles compétences en littératie ?}

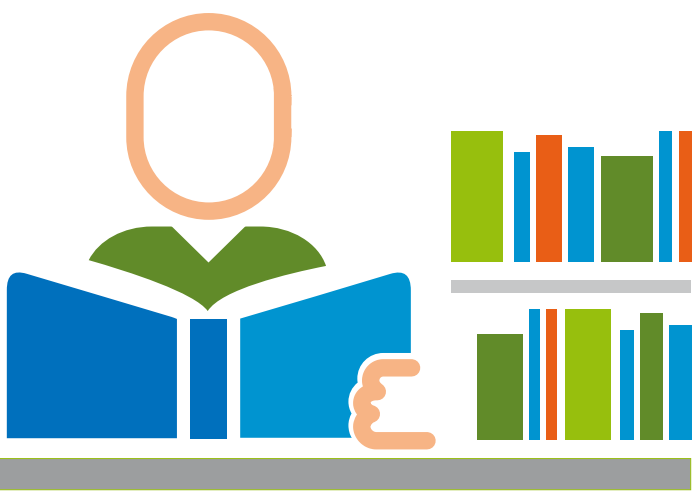

- Selon l'Évaluation des compétences des adultes, même les adultes ayant le plus faible niveau de compétences en littératie possèdent certaines compétences de base en lecture, bien que le niveau de ces dernières varie considérablement entre les pays.

- Les compétences de base en lecture se font jour à la fois dans l'exactitude et la vitesse de la réponse aux tâches de lecture - deux indicateurs du degré d'aisance et d'automaticité de la lecture.

- Parmi les adultes peu compétents en littératie, ceux passant l'évaluation dans une autre langue que leur langue maternelle affichent un niveau bien plus faible de compétences de base en lecture que les locuteurs natifs de la langue de l'évaluation.

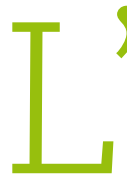

$\int$ Évaluation des compétences de adules, lancée dans le cadre du Programme de l'OCDE pour l'évaluation internationale des compétences des adultes (PIAAC),

définit la littératie comme la capacité de comprendre, d'évaluer, d'utiliser et de s'engager dans des textes écrits pour participer à la société, accomplir ses objectifs et développer ses connaissances et son potentiel. Ses résultats indiquent l'existence d'une corrélation entre la maîtrise des compétences en littératie et de nombreuses retombées positives sur la vie des individus : ceux ayant un meilleur niveau de compétences en littératie sont ainsi : moins susceptible d'être au chômage ; mieux rémunérés lorsqu'ils occupent un emploi ; et plus susceptibles d'indiquer être en bonne santé, participer à des activités bénévoles, faire confiance à autrui et estimer que leurs semblables ont leur mot à dire sur les actions des pouvoirs publics.

\section{Dans de nombreux pays, une part importante de la population adulte a un niveau faible, voire très faible, de compétences en lecture.}

Les pays pourraient tirer un avantage certain de la mise en œuvre de politiques et de programmes visant à accroître le niveau de compétences de leurs populations. Pour des raisons d'équité et d'efficacité, la priorité devrait être donnée aux programmes ciblant les adultes présentant un niveau particulièrement faible de compétences dans les fondamentaux, tels que la littératie. L'Évaluation des compétences des adultes est conçue pour analyser la totalité du spectre des compétences en littératie. Dans les 24 pays ayant participé à l'édition 2012 de cette évaluation, environ 15.5 \% des adultes âgés de 16 à 65 ans ont obtenu un faible niveau de compétences en littératie: ils se situent au niveau 1 de l'échelle mesurant le niveau de compétences en littératie, voire en deçà (cf. encadré). Ce pourcentage va de $5 \%$ au Japon à $28 \%$ en Italie. 


\section{Qu'entend-on par faibles compétences en littératie? Quels types de compétences en lecture les adultes se situant aux niveaux les plus faibles de l'évaluation possèdent-ils ?}

\section{Graphique 1 / Dans quelle mesure la maîtrise des compétences de base en lecture varie-t-elle entre les individus selon leur niveau de compétences en littératie?}
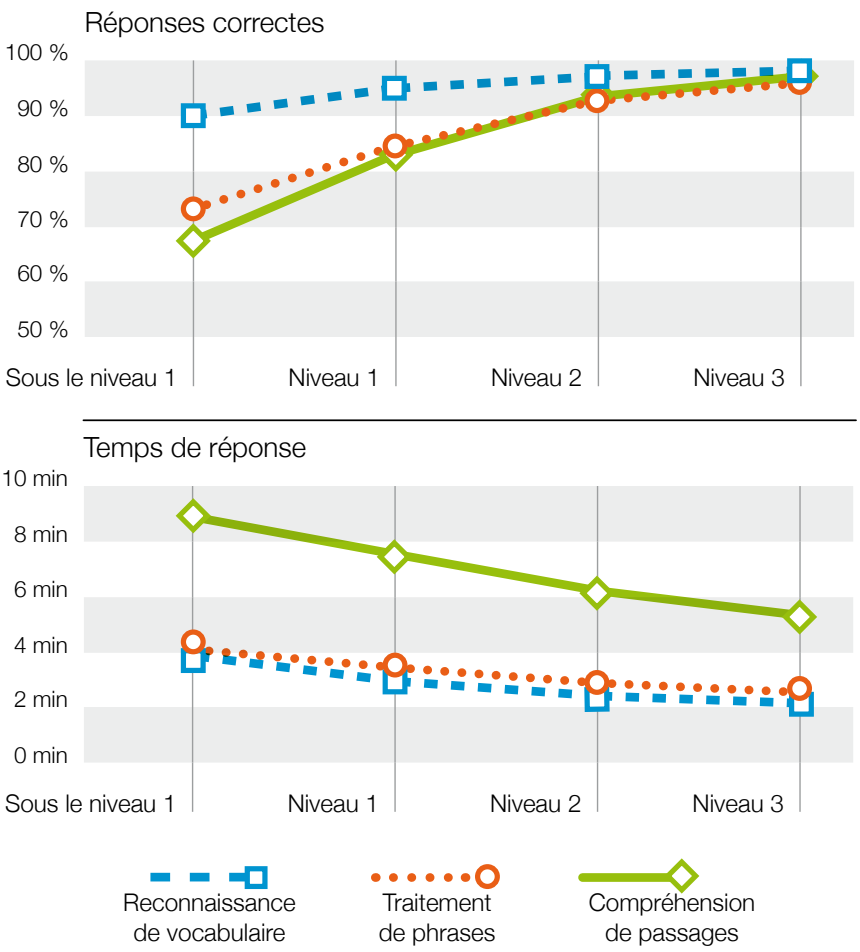

Remarque : Les niveaux se réfèrent aux niveaux de compétences PIAAC plus le niveau est élevé, plus la maîtrise des compétences est grande.

Source : OCDE (2012), Évaluation des compétences des adultes (PIAAC), www.oecd.org/site/piaac/publicdataandanalysis.htm.
Pour répondre à ces questions, l'enquête comprend une évaluation des " composantes de lecture " afin de mesurer le degré de maîtrise des composantes de base en lecture chez les adultes peu compétents en littératie. Trois composantes sont ainsi retenues : la capacité de reconnaître des mots sur support imprimé (« reconnaissance de vocabulaire », la capacité d'extraire un sens d'une phrase (" traitement de phrases ») et la capacité de comprendre des passages de textes (" compréhension de passages »). L'évaluation est conçue pour mesurer l'exactitude des réponses ainsi que le temps pris par les répondants pour mener à bien les tâches, afin d'indiquer le degré d'aisance et d'automaticité dont ils font preuve dans l'utilisation de leurs compétences de base en lecture.

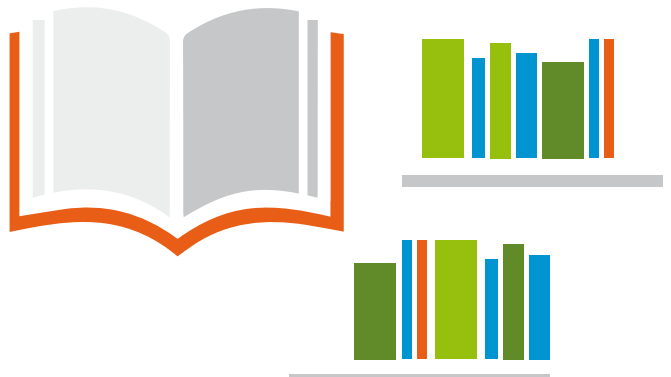

\section{Quels types de tâches les adultes peu compétents en littératie sont-ils capables d'effectuer?}

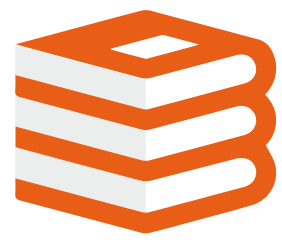

L'Évaluation des compétences des adultes définit six niveaux de compétences, du plus faible (inférieur au niveau 1) au plus élevé (niveau 5) (le niveau 1 étant celui des individus peu compétents). Les informations relatives aux réponses des participants aux différentes questions permettent de décrire les types de tâches que des individus ayant obtenu différents scores à l'évaluation sont capables d'effectuer avec une probabilité plus ou moins grande de succès. Ainsi, les adultes se situant au niveau 1 sont en général capables d'effectuer avec succès des tâches de lecture nécessitant la connaissance d'un vocabulaire de base, la compréhension du sens de phrases courtes et la lecture de paragraphes d'un texte. Ils peuvent également parfois réussir des tâches plus complexes, telles que celles nécessitant la lecture de textes plus longs et complexes, et l'utilisation de stratégies cognitives plus complexes et variées. Les adultes se situant en deçà du niveau 1 sont quant à eux censés être uniquement capables de lire des textes courts sur des sujets familiers et d'y localiser des informations concrètes et bien définies. 


\section{La compréhension de textes longs est particulièrement difficile pour les adultes les moins compétents en littératie.}

La quasi-totalité des adultes peu compétents en littératie possèdent des connaissances de base en reconnaissance de vocabulaire. Ainsi, lorsqu'on leur présente une image d'un objet familier (tel qu'un oiseau, un cercle ou une chaise), ils sont capables d'identifier le terme correspondant à cet objet dans une liste de quatre réponses possibles. En moyenne, les adultes se situant en deçà du niveau 1 sont capables d'identifier le terme correct dans $90 \%$ des cas, tandis que ceux se situant au niveau 1 y parviennent dans $95 \%$ des cas. En outre, les adultes se situant au niveau 1 effectuent généralement les tâches de reconnaissance de vocabulaire bien plus rapidement que ceux se situant en deçà de ce niveau. Le temps moyen de réponse aux tâches de reconnaissance de vocabulaire (34 tâches) s'établit ainsi à 3 minutes parmi les adultes se situant au niveau 1, contre 4 minutes parmi ceux se situant en deçà de ce niveau.

La plupart des adultes se situant au niveau 1 de compétences en littératie ou en deçà sont également capables de comprendre le sens de phrases composées d'environ 14 à 18 mots et pouvant comprendre plusieurs propositions et présenter des liens logiques complexes. Lorsqu'on leur présente une phrase en leur demandant d'indiquer si elle est logique, les adultes se situant en deçà du niveau 1 répondent correctement dans $73 \%$ des cas, en moyenne, tandis que ceux se situant au niveau 1 y parviennent dans $85 \%$ des cas. Le temps de réponse pour chaque tâche est également différent entre ces deux groupes : le temps moyen de réponse aux tâches de traitement de phrases (22 tâches) s'établit ainsi à 3.4 minutes parmi les adultes se situant au niveau 1, contre 4.2 minutes parmi ceux se situant en deçà de ce niveau.

Les adultes se situant au niveau 1 de compétences en littératie sont capables de lire des passages de texte relativement aisément, tandis que ceux se situant en deçà de ce niveau ont du mal à garder le niveau de concentration et de persévérance nécessaire pour lire des textes continus en prose comprenant plusieurs paragraphes. Les adultes de ce dernier groupe réussissent ainsi les tâches de compréhension de passages dans seulement environ $68 \%$ des cas, tandis que ceux se situant au niveau 1 y parviennent dans $84 \%$ des cas.

Graphique 2 / Prévalence des adultes peu compétents et aptitudes de ces derniers en compréhension de passages

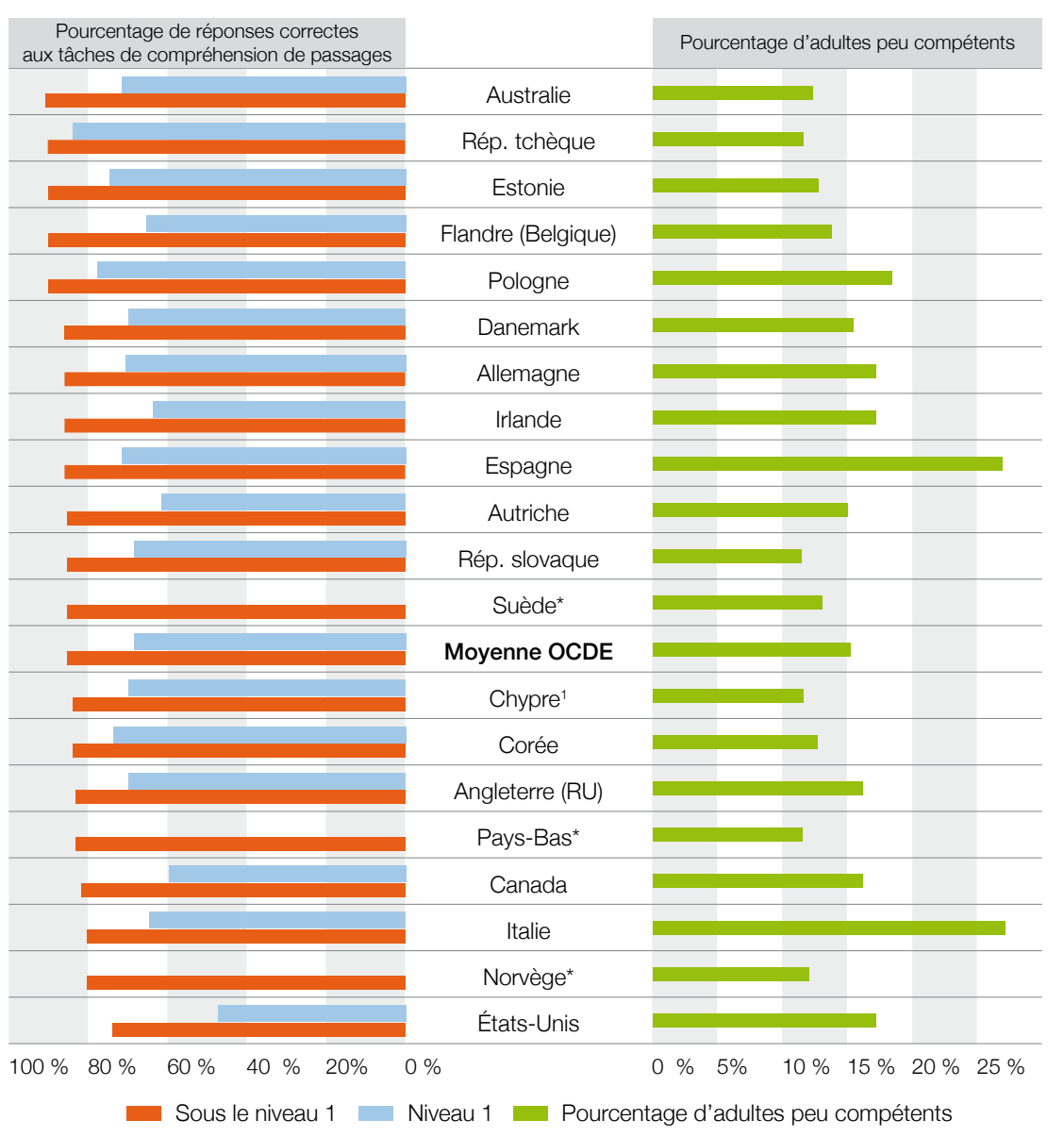

Remarques : Le panneau droit du graphique montre, pour chaque pays, le pourcentage de la population adulte ayant un faible niveau de compétences en littératie (soit les adultes se situant au niveau 1 de l'échelle PIAAC de compétences en littératie ou en deçà). Le panneau gauche du graphique montre, pour chaque pays, le pourcentage moyen de réponses correctes aux tâches de compréhension de passages parmi les adultes se situant au niveau 1 de l'échelle PIAAC de compétences en littératie ou en deçà.

Les pays sont classés par ordre décroissant du pourcentage de réponses correctes que les adultes peu compétents en littératie obtiennent aux tâches de compréhension de passages.

* Les pays suivis d'un astérisque sont ceux où le pourcentage d'adultes se situant en deçà du niveau 1 de compétences en littératie est trop faible pour être pris en compte.

1. Note de la Turquie : Les informations figurant dans ce document qui font référence à " Chypre " concernent la partie méridionale de l'lle. II n'y a pas d'autorité unique représentant à la fois les Chypriotes unique representant a la fois les Chypriotes la République Turque de Chypre Nord (RTCN). Jusqu'à ce qu'une solution durable et équitable soit trouvée dans le cadre des Nations Unies, la Turquie maintiendra sa position sur la " question chypriote".

Note de tous les États de l'Union européenne membres de l'OCDE et de l'Union européenne La République de Chypre est reconnue par tous les membres des Nations Unies sauf la Turquie. Les informations figurant dans ce document concernent la zone sous le contrôle effectif du gouvernement de la République de Chypre.

Source : OCDE (2012), Évaluation des compétences des adultes (PIAAC), www.oecd.org/site/piaac/ publicdataandanalysis.htm. 


\section{LES COMPÉTENCES DES ADULTES}

ALALOUPE

\section{Graphique 3 / Dans quelle mesure la maîtrise des compétences de base en lecture parmi les adultes peu compétents en littératie (sous le niveau 1) varie-t-elle entre les locuteurs natifs et non natifs ?}

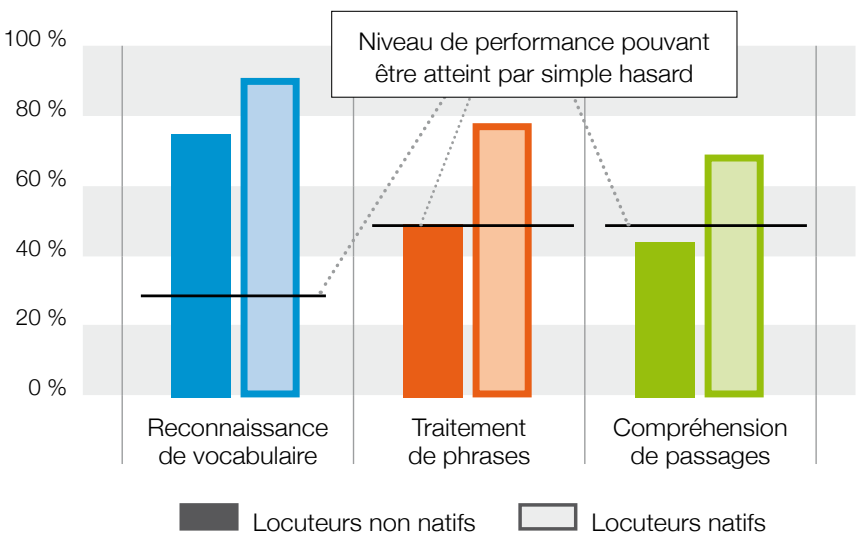

Remarque : Les tâches de traitement de phrases et de compréhension de passages ne proposant que deux possibilités de réponse, dont une correcte, les répondants ne sachant quelle réponse donner à aucune de ces tâches obtiendront tout de même environ $50 \%$ de réponses correctes en répondant au hasard. Les tâches de reconnaissance de vocabulaire proposant quatre possibilités de réponse, les répondants ne sachant quelle réponse donner à aucune de ces tâches obtiendront tout de même environ $25 \%$ de réponses correctes en répondant au hasard.

Source : OCDE (2012), Évaluation des compétences des adultes (PIAAC), www.oecd.org/site/piaac/publicdataandanalysis. htm.
Il existe des différences considérables entre les pays, non seulement en termes de nombre d'individus peu ou très peu compétents, mais également de compétences de base que ces derniers possèdent en lecture. Ainsi, aux États-Unis, les adultes les moins compétents semblent n'avoir un niveau suffisant de compétences ni en traitement de phrases, ni en compréhension de passages (avec des taux de réponses correctes de respectivement $52 \%$ et $47 \%$, proches du taux de $50 \%$ pouvant être atteint simplement par hasard). En revanche, en Corée, en Pologne et en République tchèque, les adultes les moins compétents sont capables de répondre correctement à la majorité des tâches de traitement de phrases et de compréhension de passages.

Parmi les adultes se situant en deçà du niveau 1, les différences de compétences en reconnaissance de vocabulaire, traitement de phrases et compréhension de passages sont particulièrement marquées entre les locuteurs natifs de la langue de l'évaluation et ceux qui ne le sont pas. Les locuteurs non natifs très peu compétents en littératie ont ainsi un niveau de compétences particulièrement faible lorsqu'il s'agit de comprendre des phrases et de lire des passages de textes. Il ressort de ce constat que les programmes ciblant les locuteurs non natifs devraient peut-être mettre d'abord l'accent sur l'acquisition des compétences de base en lecture avant de s'attacher au développement de compétences plus complexes.

\section{Pour
conclure \\ Pour
conclure}

Lors de l'élaboration des politiques et programmes ciblant les groupes de population peu compétents en littératie, il importe de prendre en compte les différences de niveau de maîtrise de ces compétences entre les différents groupes et au sein même de ceux-ci. Ainsi, les locuteurs natifs de la langue officielle d'un pays peuvent avoir des besoins différents de ceux des locuteurs non natifs concernant le développement de leurs compétences linguistiques ; il est par ailleurs plus difficile pour la plupart des adultes peu compétents en littératie de comprendre ce qu'ils lisent que de reconnaître des mots isolés. Enfin, le niveau de compétences de base en lecture des groupes de population peu compétents varie selon les pays ; il en va donc de même pour leurs besoins en matière de développement de leurs compétences de base en littératie. Les pays peuvent utiliser les données collectées dans le cadre de l'Évaluation des compétences des adultes afin d'affiner les dispositifs d'aide qu'ils offrent aux adultes les moins compétents.

\section{> CONTACT : $\quad$ Miloš Kankaraš (Milos.Kankaras@oecd.org) (edu.piaac@oecd.org) \\ $>$ POUR EN Grotlüschen, A. et al. (2016), " Adults with low proficiency in literacy or numeracy ", SAVOIR PLUS : Documents de travail de l'OCDE sur l'éducation, n 131, Éditions OCDE, Paris, http://dx.doi. org/10.1787/5jm0u44bnmnx-en. \\ > VISITER : Indicateurs de l'éducation à la loupe - PISA à la loupe - L'enseignement à la loupe}

Ce document et toute carte qu'il peut comprendre sont sans préjudice du statut de tout territoire, de la souveraineté s'exerçant sur ce dernier, du tracé des frontières et limites internationales, et du nom de tout territoire, ville ou région.

Vous êtes autorisés à copier, télécharger ou imprimer du contenu OCDE pour votre utilisation personnelle. Vous pouvez inclure des extraits des publications, des bases de données et produits multimédia de l'OCDE dans vos documents, présentations, blogs, sites Internet et matériel d'enseignement, sous réserve de faire mention de la source OCDE et du copyright. Les demandes pour usage public ou commercial ou de traduction devront être adressées à rights@oecd.org. 